\title{
Miniature electrostatic electron energy analyzers and S-shaped deflector
}

\author{
J. F. Williams, ${ }^{1,2}$ X. Chen, ${ }^{3}$ and P. Wilkie ${ }^{1,2}$ \\ ${ }^{1}$ Centre for Atomic, Molecular and Surface Physics, University of Western Australia, Nedlands, Perth, \\ Western Australia 6009, Australia \\ ${ }^{2}$ ARC Centre of Excellence for Antimatter-Matter Studies, University of Western Australia, Nedlands, Perth, \\ Western Australia 6009, Australia \\ ${ }^{3}$ Hefei National Laboratory for Physical Sciences at Microscale and Department of Modern Physics, \\ University of Science and Technology of China, Hefei, Anhui, 230026, China
}

(Received 29 November 2007; accepted 23 January 2008; published online 21 February 2008)

\begin{abstract}
An instrument has been developed to avoid the rotation of large electron sources and detectors in quantum single particle scattering experiments. The rotation of an electron beam has been achieved by combining three small cylindrical electrostatic electron energy analyzers in series such that the first analyzer is fixed and the other two rotate together around the exit axis of the first; it is a development from an S-shaped deflector used by Hegemann et al. [J. Phys. B 26, 4607 (1993)]. Novel design and construction, using copper, aluminum, and stainless steel parts mounted on polyvinyl printed circuit board, enabled an efficient, small-sized high vacuum compatible instrument. The characteristics and versatility of the instrument have been shown by measurements of angular and spin asymmetries of electron scattering phenomena. (c) 2008 American Institute of Physics. [DOI: 10.1063/1.2844602]
\end{abstract}

\section{INTRODUCTION}

Studies of charged particle scattering from atoms and molecules in single collision conditions cover a wide range of atomic structure and quantum scattering dynamics and frequently require a wide range of instrumentation. Presently, our studies concern the observation of asymmetries in electron spin and in angular distributions of electrons after the scattering of polarized electrons from free gaseous atoms or surfaces. The instruments required for these observations include, for example, polarized electron sources, ${ }^{1}$ moderated low energy positron sources, ${ }^{2}$ detectors of electron spin, ${ }^{3}$ and low energy electron energy analyzers. ${ }^{4}$ However, their shape, size, and weight impose limitations on the optimum design and performance of some experiments, particularly when rotation of components is preferred. These limitations are prohibitive when such instruments must be rotated, and over large angular ranges, in order to obtain data from which physically meaningful empirical models may be developed. This paper reports an instrument which $\operatorname{arose}^{5}$ from the simple idea of rotating the beam rather than a large and heavy Mott detector when measuring the angular dependence of the degree of spin polarization of a scattered electron beam. Subsequently, we found that the new instrument is more versatile and useful for a broad range of observations of elastic and inelastic electron scattering processes, ${ }^{6}$ as described below.

\section{DESIGN}

The rotational motion imposed on the beam is visualized by considering a right-handed normal rectilinear $(x, y, z)$ coordinate system with a circular beam path in the $x z$ plane with a radius $r$ and center at $(r, 0, r)$. Reflection of the semicircle with $x$ coordinates greater than $r$ in the $(x+2 r)$ axis forms an S-shaped coplanar path. Then, keeping stationary the lowest quadrant, i.e., with $0<z<r$ coordinates, and rotating the other three quadrants, as a single curve, about the $z$ axis enables the tip of the $\mathrm{S}$ shape to trace out a circular path in the $x, y$ plane around the $z$ axis. In this way, a beam after traversing the first fixed quadrant path traces a coplanar path which can rotate through any angle up to $360^{\circ}$ and which always emerges directed toward, and normal to, the $z$ axis. The realization of this simple beam path was achieved with $90^{\circ}$ and $180^{\circ}$ bends for electrons using standard cylindrical electrostatic electron energy spectrometers with input and output optics and fringing field correctors. ${ }^{7}$ Figure 1 shows a sectioned cutaway view of the instrument with the major parts indicated in a detailed caption. The rotatable $270^{\circ}$ section, with $90^{\circ}$ and $180^{\circ}$ analyzers, has been rotated about $30^{\circ}$ in Fig. 1 to enable the component parts to be seen. Each analyzer has inner and outer radii of 13.4 and $20 \mathrm{~mm}$, respectively, and their input and output optics, together with electrostatic deflection plates, are located in the parts labeled (b) and (g). Each set of input and output optics is composed of two sets of three aperture lenses composed of $0.1 \mathrm{~mm}$ thick copper sheet, spaced $1 \mathrm{~mm}$ apart with $1 \mathrm{~mm}$ diameter central beam holes. All surfaces which are exposed to the beam are coated with carbon black to reduce stray electron signals.

This combination of the three electron energy analyzers to achieve rotation also gave flexibility for the ways in which they could be used for electron energy analysis. We chose to use the fixed first $90^{\circ}$ analyzer as a "preanalyzer" and its output image at plate (d) (see Fig. 1) as the input image for the $270^{\circ}$ analyzer. The final image was at the output plane of the $270^{\circ}$ analyzer. The beam optics were modeled using SIMION. The success of this approach is shown by the observations described later.

A novel feature is the miniaturization and mounting of the individual parts of the instrument on printed circuit board 


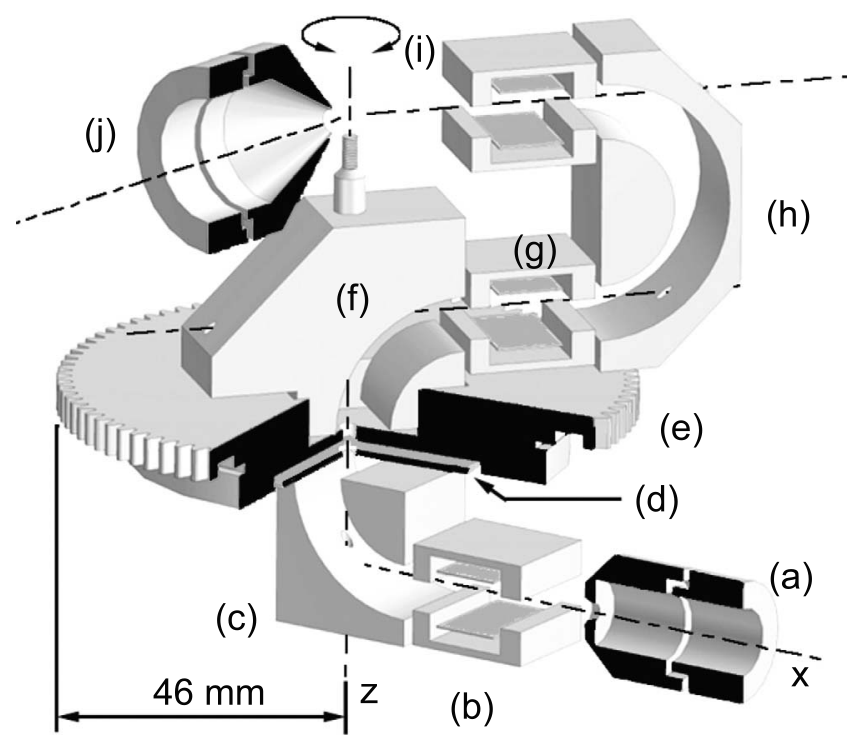

FIG. 1. A sectioned view of the basis of the S-bender instrument shows the three electron energy analyzers (c), (f), and (h) with entrance and exit optics (b) and (g) (only some shown). The entrance (first) analyzer (c) is fixed and the other two analyzers (f) and (h) rotate together on a platform (e) around the normal emergent axis, the $z$ axis, from the entrance analyzer. The axes $x$ and $z$ form part of a normal rectilinear $(x, y, z)$ set. The exit beam from the first analyzer passes through two sequential apertures, represented as (d), before entering analyzer (f). The emergent beam from the $\mathrm{S}$ bender lies in a plane parallel to the $x y$ plane and is shown with a rotation around the $z$ axis of about $30^{\circ}$ relative to the input beam on the $x$ axis. The arrow (i) indicates rotation around a long thin tube which forms the target gas jet. The electrons scattered in the $x y$ plane are shown entering a detector $(\mathrm{j})$ at some other angle in the $x y$ plane. The figure is drawn to scale. The inner and outer radii are 13.4 and $20 \mathrm{~mm}$, respectively.

(PCB). This construction enabled simple electrical connection of the copper components with increased reliability and minimization of the size of the instrument. The initial construction was intended for use with base vacuum pressures of about $10^{-8}$ Torr and used standard epoxy and polyphenylene oxide resin PCB. This reported version has a polyimide PCB, is bakable to at least $130{ }^{\circ} \mathrm{C}$ with good mechanical stability and has good ultrahigh vacuum characteristics with a base pressure of $7 \times 10^{-10}$ Torr. Electrical leads were connected via Kapton (polyimide)-covered stainless steel wire.

All materials were measured to be nonmagnetic to less than $10 \mathrm{mG}$. Because the instrument was designed to transport spin-polarized electrons through the rotatable $270^{\circ}$ section, it is important that the residual magnetic field gradient was negligibly small so that the direction of the electron spin remained constant.

This combination of electron energy analyzers was used in high or low resolution electron energy modes. In a high resolution mode with the energy resolution set at $50 \mathrm{meV}$, for example, the scattered electrons, from either elastic or inelastic scattering events, were selected by scanning the analyzer transmission energy across the peak. Using all three of the energy analyzers achieved an electron energy resolution of about $16 \mathrm{meV}$ for a final beam energy of about $0.25 \mathrm{eV}$. In a low energy resolution mode, or equivalently a high transmission mode, the analyzers were operated with a wide energy passband, such that a flat-topped transmitted beam profile was obtained.
The S-bender instrument has been used in so-called normal and inverse modes according to the direction of transit of the beam. In the normal mode, an incident beam approached from the right hand side along the $x$ axis, as in Fig. 1, traversed the $\mathrm{S}$ bender, interacted with a gaseous target after which the scattered electrons were selected, for example, by an electron spin polarization analyzer, usually a large nonrotatable Mott detector. In such cases, the $\mathrm{S}$ bender was used to rotate the incident electron beam relative to the angular location of the Mott detector. In the inverse mode, a high energy electron beam emerged from the last optical element of its source, as for example element (j) in Fig. 1, and interacted with the target atoms. Then, for example, when detecting low energy ejected electrons, the entrance aperture of the $\mathrm{S}$ bender selects electrons at some angle and transports them to exit through the optics (b) of Fig. 1 and into a fixed detector, which could be a Mott detector. In both modes, the rotation of a very large source and/or detector, with separate vacuum pumping system, was avoided. While these modes are similar, their experimental implementations, operating potentials, and use can be quite different. This paper now describes the various uses and measurements of scattering probabilities, and spin asymmetries, for various collision processes, which have demonstrated the successful and useful performance of the instrument.

\section{PERFORMANCE}

An essential characteristic of the instrument when used in a low energy resolution mode was that it transmitted, with as little modification as possible, the characteristics of the input beam. The success of SIMION ray tracing for design of the electron optical characteristics and of the subsequent experimental optimization, as well as of the complete design and construction and operation of the instrument were shown, within the experimental uncertainties, by measuring well-known elastic electron scattering cross sections. The same measurements also tested the effect of the $\mathrm{S}$ bender on the angular resolution of the electron beam by observing the angular elastic scattering of $40 \mathrm{eV}$ electrons from argon atoms in the vicinity of its deep minima near $68^{\circ}$ and $142^{\circ}$. The observed data were normalized to the data of Williams and Willis ${ }^{4}$ to provide the elastic angular differential cross sections, as shown in Fig. 2. Two aspects need comment. First, the measurements were made for left and right scattering angles, i.e., on both sides of the unscattered electron beam, by using the deflector to provide effectively rotation of (a) the scattered electron energy spectrometer and of (b) the incident electron beam. Note that these two tests involved considerable change in the experimental apparatus, as outlined above. Usually, for measurements of electron scattering phenomena, only one of these procedures is used but here, we used both procedures to test the instrument. The two data sets agreed within statistical uncertainties and are shown by their average values in Fig. 2. The data are in excellent agreement with values calculated from the convergent close coupling (CCC) theory, ${ }^{8}$ which are not shown for clarity. The angular dips near $68^{\circ}$ and $142^{\circ}$, where the cross section varies rapidly over a narrow angular range of about $10^{\circ}$, indi- 


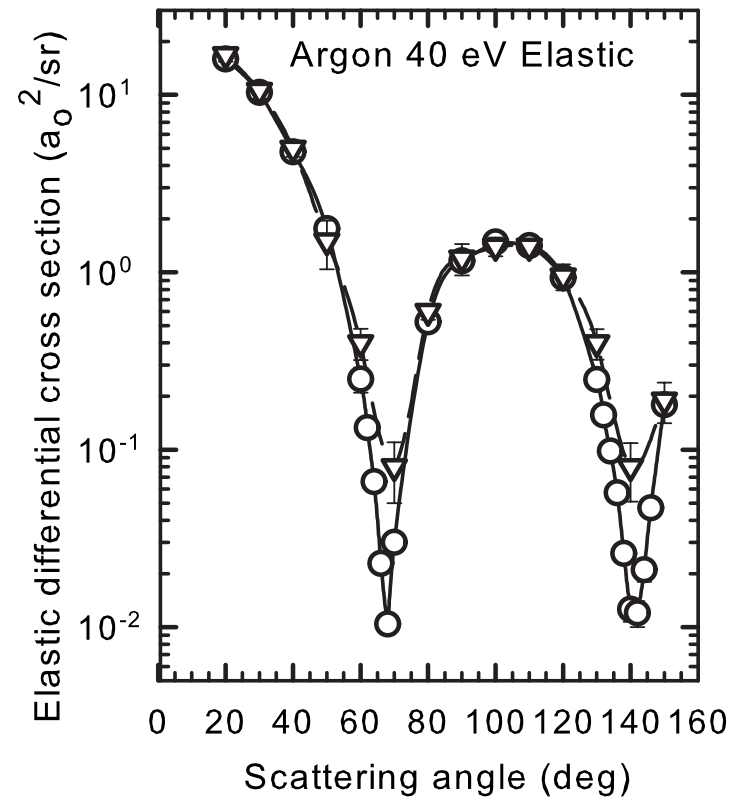

FIG. 2. The elastic differential cross section of argon for $40 \mathrm{eV}$ incident electron energy. The measured data are shown by inverted open triangles and are the averages of data obtained by (a) rotating the scattered electron energy analyzer and (b) by rotating the incident electron beam which were coincident within the measured uncertainties. The data of Ref. 4 are shown for comparison. The lines are best polynomial fits to the data.

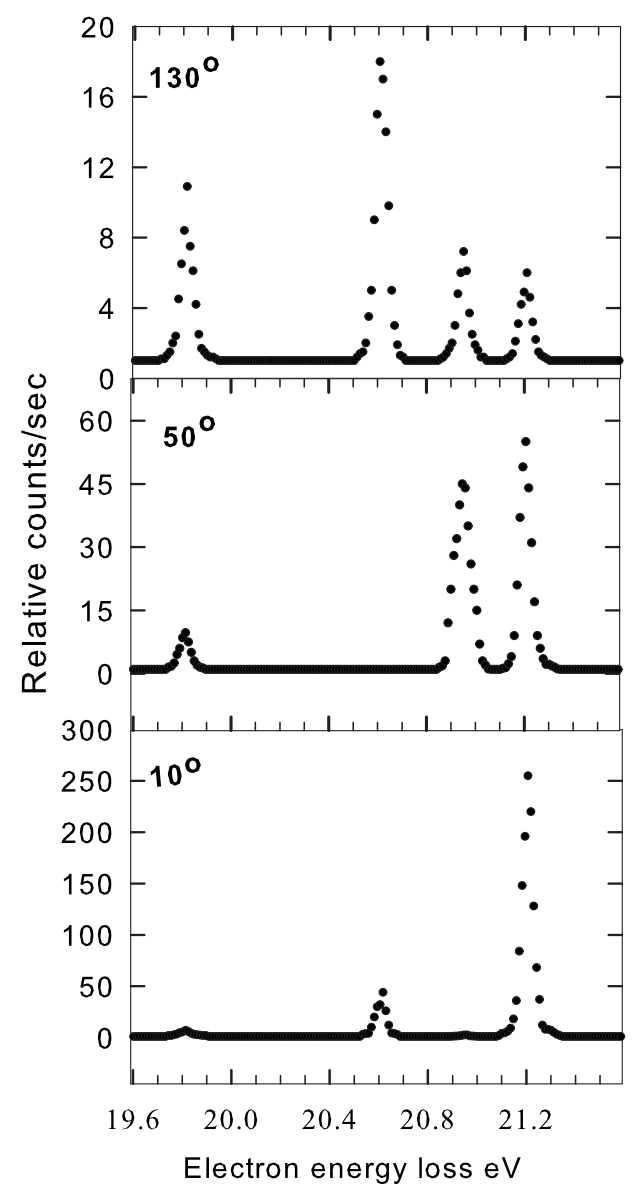

FIG. 3. For $40 \mathrm{eV}$ electrons incident on helium, the $2^{3} S(19.81 \mathrm{eV}), 2^{1} S$ $(20.61 \mathrm{eV}), 2^{3} P(20.96 \mathrm{eV})$, and $2^{1} P(21.21 \mathrm{eV})$ states are shown as a function of electron energy loss at electron scattering angles of $10^{\circ}, 50^{\circ}$, and $130^{\circ}$ in the lower, middle, and upper sections, respectively.

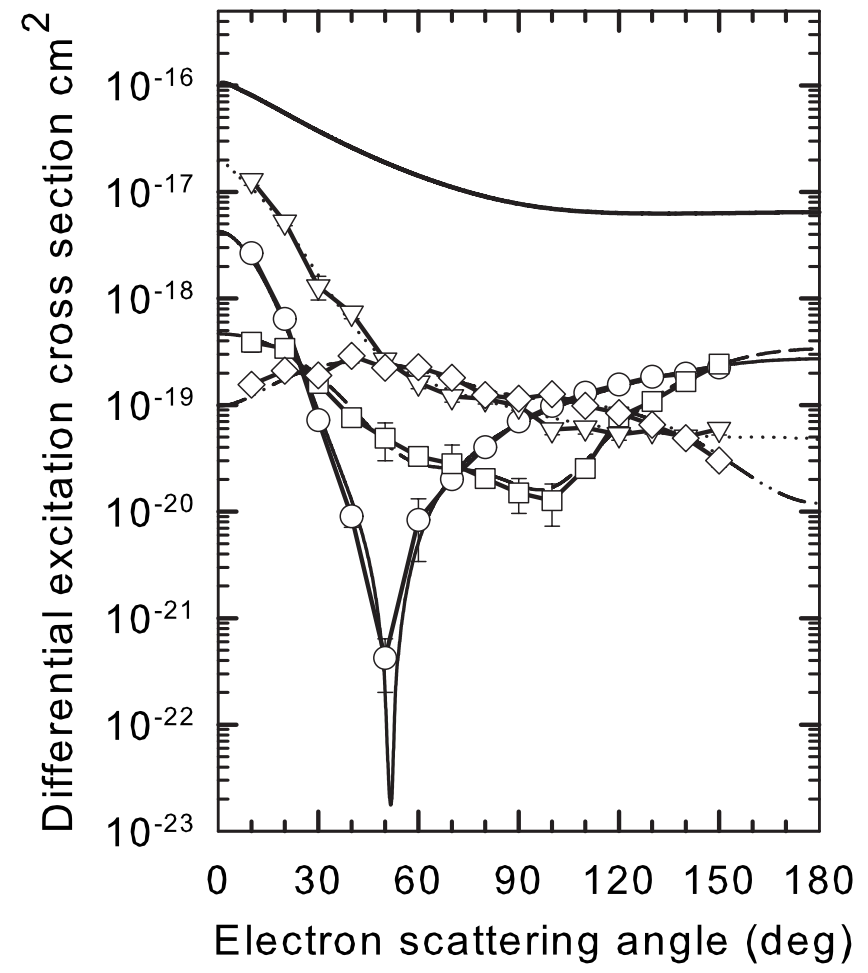

FIG. 4. The angular differential excitation cross sections for the $2^{1} S, 2^{3} S$, $2^{3} P$, and $2^{1} P$ states of helium for $40 \mathrm{eV}$ incident electrons. The data are derived from observations such as in Fig. 3 and are calibrated to the convergent close coupling theoretical values for the $2^{1} P$ state at $21^{\circ}$ scattering angle (Ref. 8) of $4.77 \times 10^{-18} \mathrm{~cm}^{2}$. The data for the $2^{1} S, 2^{1} P, 2^{3} S$, and $2^{3} P$ states are shown as open circles, inverted triangles, squares, and diamonds, respectively. The full lines are the theoretical convergent close coupling data from Ref. 8, the top line is the elastic scattering cross section.

cated an angular resolution of $3^{\circ}$ which was accepted at that stage of development. Also, angular distribution data were obtained with the $\mathrm{S}$ bender acting as a deflector for both very narrow $50 \mathrm{meV}$ and very wide $750 \mathrm{meV}$ energy spreads of the transmitted electron beam to ensure that the deflector did not change the energy resolutions.

Figure 3 shows, for $40 \mathrm{eV}$ electrons incident on a gaseous helium atom beam, the $2^{1} \mathrm{~S}, 2^{3} \mathrm{~S}, 2^{3} \mathrm{P}$, and $2^{1} \mathrm{P}$ state energy loss peaks at electron scattering angles of $10^{\circ}, 50^{\circ}$, and $130^{\circ}$. These energy loss features were studied because an incident electron energy resolution of about $50 \mathrm{meV}$ separated those states, their relative excitation functions could be determined from a single $2 \mathrm{eV}$ scan of the energy loss region and their cross section values were known to span about five orders of magnitude. ${ }^{8}$ The combination of these features presented an excellent variation of physical parameters which could be used simultaneously to test the performance of the instrument. For example, the absence of the $2^{1} S$ peak at $50^{\circ}$ compared with that at $130^{\circ}$ and $10^{\circ}$ enabled a check on the suppression of background electrons for those particular operating conditions. The phenomenology of these relative energy loss features led to further measurements for helium with $40 \mathrm{eV}$ incident electrons and over a range of scattering angles to determine the ratios of the areas under the energy loss peaks for the $2^{1} \mathrm{~S}, 2^{3} \mathrm{~S}$, and $2^{3} \mathrm{P}$ states to the area under the peak for the $2^{1} P$ state. The relative values were normalized to the CCC calculated values for the $2^{1} \mathrm{P}$ state at $21^{\circ}$ to 


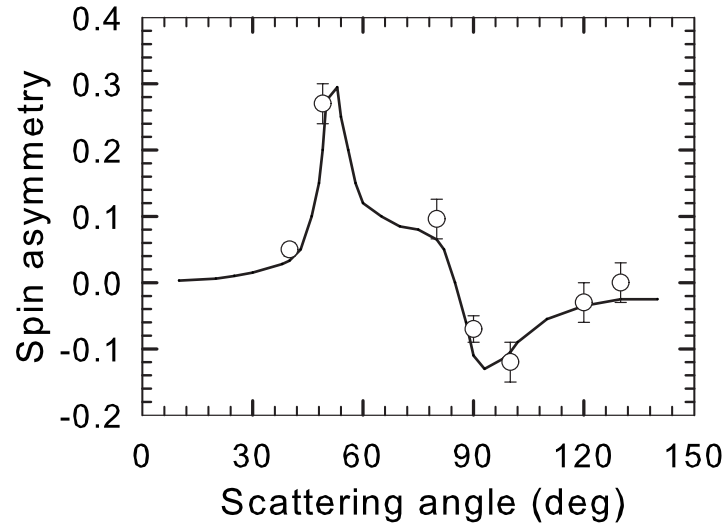

FIG. 5. The electron asymmetry factor $S_{A}$ for left and right angular elastic scattering from xenon atoms for $40 \mathrm{eV}$ incident electrons as a function of electron scattering angle. The present data are shown as open circles and the theoretical values from Ref. 10 are shown as a full line.

provide calibrated cross section values. Figure 4 shows the angular differential excitation cross sections for the $2^{1} S, 2^{3} S$, and $2^{3} P$ states of helium at $40 \mathrm{eV}$. As mentioned above, the large variations of these cross sections and of their relative values made their observation ideal for checking the behavior of the instrument for linear response across a given energy range, uniform angular response, and level of background electrons, for example. These tests were an iterative process between measurement, plotting the angular dependence of the relative values of peak areas, comparing with the $\mathrm{CCC}$ calculated values ${ }^{8}$ and making instrumental changes if required. The "correct" relative heights of the spectral peaks could only be assessed against some regular behavioral dependence on angle such as offered by reliable cross section values. The outcome of these measurements is that the observable features of the energy loss spectra indicate the excellent behavior of the S-bender instrument. The measured data are in good agreement, i.e., within their statistical uncertainties, with the well-established CCC calculations. ${ }^{8}$

Finally, the performance of the instrument using a spinpolarized electron beam was tested by measuring the count rates $N_{U}$ and $N_{D}$ for the scattering of spin-up and spin-down electrons, respectively, at several scattering angles $(\theta)$ and at an energy $E$ of $40 \mathrm{eV}$. These measurements were made also with a procedure similar to that in the previous paragraph. The measurements were made for left and right scattering angles, i.e., on both sides of the unscattered electron beam, by using the deflector to provide the equivalent rotation of (a) the scattered electron energy spectrometer and of (b) the incident electron beam. This procedure involved considerable change in the experimental apparatus, as outlined above, and of each type of rotation which must, in principle, be identical.

These observed count rates are related to the Sherman function $^{9} S(\theta, E)$ given by the relation $S(\theta, E)=(1 / P)$ $\left[N_{U}-N_{D}\right] /\left[N_{U}+N_{D}\right]$, where $P$ is the degree of polarization of the incident electron beam perpendicular to the scattering plane. Figure 5 shows $S(\theta, E)$ for $40 \mathrm{eV}$ elastic scattering in xenon which was chosen because the spin-orbit interaction is large for large atomic number atoms and the Sherman func- tion values are large compared with those of lighter atoms. The measurements were made after the above characterizations were made. First, the function shows sharp angular structure at intermediate energies in xenon ${ }^{10}$ and, consequently, is sensitive to the magnitude of the instrumental resolution which can broaden an angular response. Second, it is the nature of quantum scattering that the Sherman function has minima and maxima values which usually occur within small angular ranges where the unpolarized elastic differential cross section is small. Consequently, after the satisfactory performance of the deflectors for angular and energy resolution were established, the Sherman function could be measured reliably with increased certainty. The magnitude of $S(\theta, E)$ is in good agreement within experimental uncertainties with previous data ${ }^{10}$ and calculated values ${ }^{10}$ using a Dirac-Fock approach with a polarization potential. Hence, for these scattering parameters, there is increased confidence that this approach is a good approximation to the real observed scattering process within the measured uncertainties.

\section{CONCLUSIONS}

Many tests using a wide variety of energy and angular resolutions, and geometrical configurations, for several elastic and inelastic differential cross sections and their ratios have shown that the S-bender combination of electron energy analyzers has an excellent performance for low energy electron scattering measurements. Measurements of the Sherman function in xenon have combined those features with analysis of electron spin observations to indicate the suitability of the instrument for future observations of spin-dependent quantum scattering structure and dynamical measurements. The use of the instrument can be extended to rotate any charged particle beam, for example, positron, positive or negative ion, and similarly to direct the selected electrons or charged ions produced by a scattering event into a nonrotatable detector.

\section{ACKNOWLEDGMENTS}

Professor Chen was supported by an Australian Academy of Science Fellowship. The research was supported by the Australian Research Council and the University of Western Australia. The mechanical workshop in the Physics Department at the University of Western Australia made this instrument possible.

${ }^{1}$ D. T. Pierce, R. J. Celotta, G. C. Wang, W. N. Unertl, A. Galejs, C. E. Kuyatt, and S. R. Mielczarek, Rev. Sci. Instrum. 51, 478 (1980).

${ }^{2}$ N. G. Fazleev, A. H. Weiss, and J. L. Fry, Mater. Sci. Forum 445, 483 (2004).

${ }^{3}$ V. N. Petrov, M. S. Galaktionov, and A. S. Kamochkin, Rev. Sci. Instrum. 72, 3728 (2001)

${ }^{4}$ J. F. Williams and B. A. Willis, J. Phys. B 8, 1670 (1975).

${ }^{5}$ T. Hegemann, S. Schroll, and G. F. Hanne, J. Phys. B 26, 4607 (1993).

${ }^{6} \mathrm{~N}$. Andersen and K. Bartschat, Polarization, Alignment, and Orientation in Atomic Collisions (Springer, New York, 2001)

${ }^{7}$ P. Hayes, M. A. Bennett, J. Flexman, and J. F. Williams, Rev. Sci. Instrum. 59, 2445 (1988)

${ }^{8}$ D. Fursa and I. Bray, Phys. Rev. A 52, 1279 (1995).

${ }^{9}$ J. Kessler, Polarized Electrons, 2nd ed. (Springer, Berlin, 1985).

${ }^{10}$ A. Dorn, A. Elliott, J. Lower, S. F. Mazevet, R. P. McEachran, I. E. McCarthy, and E. Weigold, J. Phys. B 31, 547 (1998). 
Review of Scientific Instruments is copyrighted by the American Institute of Physics (AIP). Redistribution of journal material is subject to the AIP online journal license and/or AIP copyright. For more information, see http://ojps.aip.org/rsio/rsicr.jsp 